\title{
3-Colorability of Pseudo-Triangulations
}

\author{
Oswin Aichholzer, Franz Aurenhammer, Thomas Hackl, \\ Clemens Huemer, Alexander Pilz, Birgit Vogtenhuber
}

\begin{abstract}
Deciding 3-colorability for general plane graphs is known to be an NPcomplete problem. However, for certain families of graphs, like triangulations, polynomial time algorithms exist. We consider the family of pseudotriangulations, which are a generalization of triangulations, and prove NP-completeness for this class. This result also holds if we bound their face degree to four, or exclusively consider pointed pseudo-triangulations with maximum face degree five. In contrast to these completeness results, we show that pointed pseudo-triangulations with maximum face degree four are always 3-colorable. An according 3-coloring can be found in linear time. Some complexity results relating to the rank of pseudotriangulations are also given.
\end{abstract}

\section{Introduction}

Graph coloring is an important problem in discrete mathematics, with many applications [8]. A (proper) $k$-coloring of an undirected graph $G$ is an assignment of colors to the vertices of $G$, one out of $k$ colors for each vertex, such that adjacent vertices get assigned different colors. Of course, if $k$ is chosen too small, or $G$ is too dense, a $k$-coloring will not exist. Deciding $k$-colorability of a given graph class can be an intriguing problem.

We will concentrate on plane geometric graphs in this paper, more specifically, on planar straight-line graphs, and $k=3$. Deciding 3-colorability of a given planar graph is known to be NP-complete [14]. For triangulations, 3 -colorability can be decided in linear time, as it is necessary and sufficient that every inner vertex has even degree. Alternatively, we can use the following constructive algorithm: Start with the three different colors of one triangle. Then the color of the third vertex of each neighboring (edge-adjacent) triangle is determined. This process is iterated until either we obtain a proper coloring or a contradiction occurs (an already colored vertex is forced to have a different color), in which case obviously no proper 3-coloring exists.

It is natural to ask for which other types of plane geometric graphs the decision problem can be solved efficiently. Trivially, 3-colorings always exist for trees, cycles, and quadrilateralizations. Maximal outerplanar graphs (or equivalently, triangulations of simple polygons, or triangulations of point sets in convex position) are also 3-colorable. Ellingham et al. [6] and Diks, Kowalik, and 


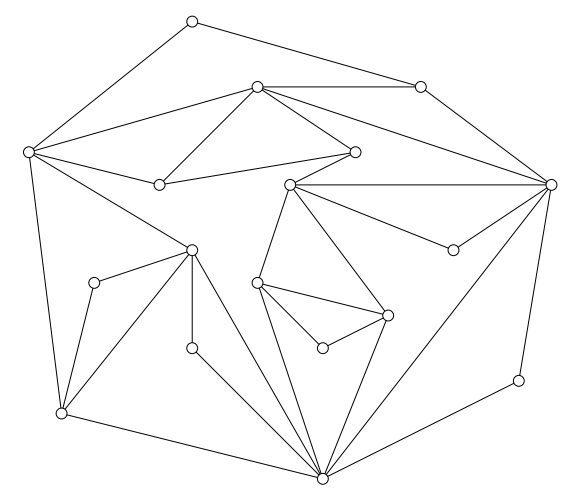

Figure 1: A pointed pseudo-triangulation

Kurowski [5] give a characterization of plane graphs with isolated non-triangular faces that are 3-colorable. Moreover, 3-colorability is linear-time decidable for general locally connected graphs [9]. ${ }^{1}$ See [13] for a survey on 3-colorability.

In this work we consider so-called pseudo-triangulations, which generalize triangulations in several aspects. They constitute a natural choice of a family of graphs which is more complex than trees or triangulations, but still rather specific. A pseudo-triangulation is a planar straight-line graph where each face (except the outer one) is a pseudo-triangle, that is, a polygon with exactly three convex interior angles. ${ }^{2}$ See Figure 1 for an illustration. Pseudo-triangles can be large, containing many vertices with reflex interior angles. Triangulations are the special case where no reflex angles occur in the inner faces. Pseudotriangulations are versatile structures with many applications, and with interesting combinatorial and geometric properties; see, e.g., [1, 12].

A vertex $v$ of a planar straight-line graph $G$ is called pointed if (exactly) one of its incident faces has a reflex angle at $v$. Similarly, if all vertices of $G$ are pointed then we talk of a pointed planar straight-line graph. Pointed pseudotriangulations (see Figure 1 again) are of particular interest in this paper. They realize the minimum number of edges, $2 n-3$, among all possible pseudotriangulations (for a given set of $n$ vertices), and are known to be minimally rigid graphs [15]. Moreover, 'almost pointed' pseudo-triangulations (i.e., those with small rank; see Section 2) are generalized planar Laman graphs and, as such, have special edge and face intersection properties [2].

We show that the problem of deciding whether a pseudo-triangulation is 3 -colorable is NP-complete. To this end we prove that, with respect to 3coloring, any geometric straight-line graph can be reduced to a (pointed) pseudotriangulation. For the special case of pointed pseudo-triangulations with con-

\footnotetext{
${ }^{1} \mathrm{~A}$ graph is locally connected if, for every vertex $v$, the subgraph induced by its neighbors (not including $v$ ) is a connected graph. In particular, this is the case for triangulations.

${ }^{2}$ Note that the embedding of a graph does not influence whether it is 3 -colorable. We use the straight-line embedding mainly to define the families of graphs considered here. Further, the embedding can reveal certain combinatorial properties.
} 
stant maximum face degree, we obtain that the problem remains NP-complete if the degree bound is at least five. As a complementary result, it is demonstrated that for pointed pseudo-triangulations with maximum face degree 4 , a 3 -coloring always exists and can be found in linear time.

Throughout this paper, we assume that the vertex sets of the graphs to be considered are in general position, that is, no three vertices lie on a common straight line.

\section{2 (Pointed) Pseudo-Triangulations}

In this section, we start with a colorability result for general planar straight-line graphs which are pointed, and then draw connections to pseudo-triangulations with various 'levels of pointedness'.

For a finite planar vertex set $S$, let $n=|S|$, and denote by $|C H(S)|$ the number of extreme points of $S$ (i.e., the number of points on the boundary of the convex hull of $S$ ). The rank of a pseudo-triangulation is the number of non-pointed vertices in it; see [1] for further details. Observe that pointed pseudo-triangulations have rank zero, whereas any triangulation of $S$ attains a maximum rank, $i(S)=|S|-|C H(S)| \geq 0$.

We need the following technical lemma.

Lemma 1. Any planar straight-line graph $G(S)$ on $S$ can be extended to a pseudo-triangulation, $T\left(S^{\prime}\right)$, with vertex set $S^{\prime}$ such that:

- $S \subseteq S^{\prime}$ and $\left|S^{\prime}\right|=\Theta(n)$,

- $G(S)$ is 3-colorable if and only if $T\left(S^{\prime}\right)$ is 3-colorable,

- the rank of $T\left(S^{\prime}\right)$ equals the number of non-pointed vertices in $G(S)$.

Proof. From Theorem 2.6 in [12] it follows that, by adding a linear number of edges, any planar straight-line graph $G(S)$ can be augmented in polynomial time to a pseudo-triangulation without changing the pointedness of the graph. Here we will use, instead of single edges, the gadget depicted in Figure 2(a) to connect two vertices, in order to obtain a pseudo-triangulation $T\left(S^{\prime}\right)$.

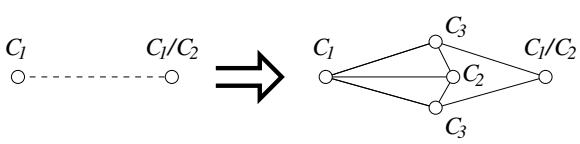

(a)

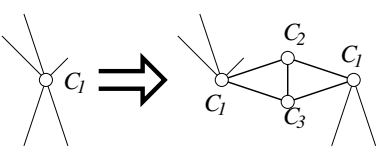

(b)

Figure 2: (a) Transforming into a pseudo-triangulation. (b) Transforming into a pointed planar straight-line graph. Colors $C_{1}$ to $C_{3}$ indicate possible color configurations.

First observe that $\left|S^{\prime}\right|=\Theta(n)$ holds, as one gadget adds only a constant number of vertices. Second, the gadgets can be added in a way such that they 
do not change the pointedness of the involved vertices: By the general position assumption on $S$, the gadgets can be made sufficiently narrow. As the new vertices introduced with each gadget are all pointed, it follows that the number of non-pointed vertices remains unchanged.

Finally, adding the gadgets does not add additional coloring restrictions on the vertices they connect. They might be colored arbitrarily (identically or differently), and still the added vertices of the gadget are 3-colorable. Thus, $G(S)$ is 3-colorable if and only if $T\left(S^{\prime}\right)$ is 3-colorable.

As Planar Graph 3-colorability is known to be NP-complete[14], Lemma 1 already implies the following NP-completeness result.

Corollary 1 (Pseudo-Triangulation 3-colorability, PT-3col). Deciding whether a pseudo-triangulation is 3-colorable is NP-complete.

Pointed pseudo-triangulations are an important subset of pseudo-triangulations. As already mentioned, they minimize the number of edges over all pseudotriangulations and thus, in some way, also the number of color restrictions. Nevertheless, we will show that even for this restricted class, deciding 3-colorability is NP-complete. To this end, we prove that Pointed Planar GraPh 3-COlorability is NP-complete, from which NP-completeness of Pointed Pseudo-Triangulation 3-COLORABILITY follows.

Lemma 2 (Pointed Planar Graph 3-colorability, PG-3col). Deciding whether a pointed planar straight-line graph is 3-colorable is NP-complete.

Proof. We show how to transform a given planar straight-line graph $G$ into a pointed planar straight-line graph $G^{\prime}$, such that $G$ is 3-colorable if and only if $G^{\prime}$ is 3-colorable. Without loss of generality, assume that there are no horizontal edges in the given embedding of $G$, as otherwise we can slightly rotate the plane. Now every non-pointed vertex $v$ of $G$ is replaced by two duplicates $v_{L}$ and $v_{R}$ of $v$. The two copies are placed sufficiently close to the left $\left(v_{L}\right)$ and to the right $\left(v_{R}\right)$ of $v$, respectively. All edges incident to $v$ from above will now be incident to $v_{L}$, and all edges incident to $v$ from below will be moved to $v_{R}$. In addition, $v_{L}$ and $v_{R}$ are connected by a small construction consisting of five edges, as shown in Figure 2(b).

By the general position assumption, the resulting graph $G^{\prime}$ is connected and planar. Only a linear number of vertices has been added, and all the vertices are now pointed. Moreover, the gadget connecting $v_{L}$ and $v_{R}$ ensures that, in a proper 3-coloring of $G^{\prime}$, both vertices have to get the same color. Thus, $G$ is 3-colorable if and only if $G^{\prime}$ is 3-colorable. NP-completeness follows, as the transformation can be done in polynomial time.

Combining Lemma 1 with Lemma 2 proves the next result.

Theorem 1 (Pointed Pseudo-Triangulation 3-colorability, PPT-3col). Deciding whether a pointed pseudo-triangulation is 3-colorable is NP-complete. 
Theorem 1 gives rise to an interesting question. On the one hand, pointed pseudo-triangulations have rank 0 and, as shown in Theorem 1, it is NP-complete to decide their 3-colorability. On the other hand, triangulations of the point set $S$ have maximum $\operatorname{rank} i(S)=|S|-|C H(S)|$, i.e., all inner vertices are nonpointed, and 3-colorability then can be decided in linear time. It is natural to ask for which rank the change from 'solvable' to 'intractable' happens. With the following two theorems, we make a first step towards answering this question.

Theorem 2. Let $k=i(S)-r$ be the number of inner pointed vertices of a pseudo-triangulation $T(S)$ of rank $r$. 3-colorability of $T(S)$ can be decided in $O\left(4^{k} \cdot n\right)$ time.

Proof. Recall from Section 1 that a triangulation can be 3 -colored by considering a spanning tree in its dual graph. We first pick an edge of the triangulation and color its vertices with colors $c_{1}$ and $c_{2}$. We then traverse the spanning tree of the dual, and in each visited triangle we color the third vertex with the remaining color. The same traversal can be applied to any pseudo-triangulation (or any planar graph). However, for a non-triangular face, we thereby only fix the two colors for the edge through which we reached the face, but might not be able to locally decide the color of the remaining vertices of that face.

Let $F$ be the set of faces of $T(S)$ which are not triangles. We denote by $p_{f} \geq 1$ the number of reflex vertices in a face $f \in F$. Then $f$ has $3+p_{f}$ vertices, and $\sum_{f \in F} p_{f}=k$. Once the vertices of an edge $e$ of a face $f$ are assigned their colors, we color the remaining vertices of $f$ in the order given by $f$, starting at $e$. For each following vertex we have (at most) two possibilities.

It is easy to see that the number of different 3-colorings of all vertices incident to faces in $F$ that we guess in this way is at most $\prod_{f \in F}\left(2^{1+p_{f}}\right)=2^{|F|+k} \leq 2^{2 k}$, as $|F| \leq k$. Since the spanning tree of the dual graph has size $O(n)$, the time bound follows by applying a straight-forward backtracking approach.

In particular, restricting the rank $r$ such that $k \in O(\log n), 3$-colorability of the pseudo-triangulation can be decided in polynomial time. Intuitively speaking, if, for any constant $0<\varepsilon \leq 1$, this restriction is relaxed to an input domain such that the rank $r$ is allowed to have any value in $i(S)-\Omega\left(n^{\varepsilon}\right)$, then the problem becomes NP-complete. This fact is stated in Theorem 3 .

Theorem 3 (RAnk $\boldsymbol{r}$ Pseudo-Triangulation 3-colorability, $\mathrm{PT}_{\boldsymbol{r}}$-3col). For any constant $0<\varepsilon \leq 1$, let $f(x) \in \Omega\left(x^{\varepsilon}\right)$. Then it remains NP-complete to decide 3-colorability of a pseudo-triangulation $T(S)$ with rank $r \geq i(S)-f(|S|)$.

Proof. To prove this theorem, we present a polynomial-time reduction from PPT-3col (which is NP-complete by Theorem 1). We show how to extend an arbitrary pointed pseudo-triangulation $T^{\prime}\left(S^{\prime}\right)$ to a rank- $r$ pseudo-triangulation $T_{r}(S), 0 \leq r \leq i(S)-\Omega\left(n^{\varepsilon}\right)$, of a point set $S$ of size $n$ such that 3 -colorability persists.

Circumscribing $T^{\prime}\left(S^{\prime}\right)$ with a triangle $\triangle$ adds 3 points to $S^{\prime}$. By Lemma 1 , we can extend the graph $T^{\prime}\left(S^{\prime}\right) \cup \triangle$ to a pointed pseudo-triangulation $T^{\prime \prime}\left(S^{\prime \prime}\right)$, 

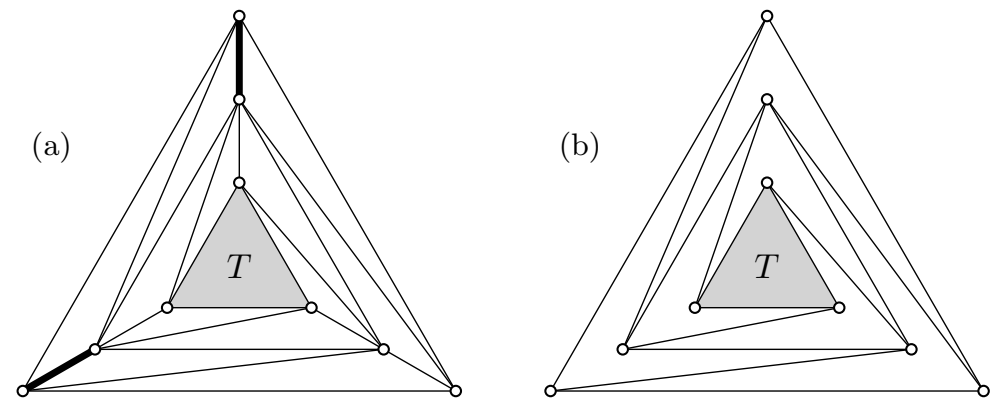

Figure 3: Constructing pseudo-triangulations with high or low rank that are 3 -colorable if and only if $T$ is 3 -colorable.

such that $\left|S^{\prime \prime}\right|=\Theta\left(\left|S^{\prime}\right|\right)$, and $T^{\prime}\left(S^{\prime}\right)$ is 3-colorable if and only if $T^{\prime \prime}\left(S^{\prime \prime}\right)$ is 3 -colorable.

We increase the number of non-pointed vertices of our construction by extending $T^{\prime \prime}\left(S^{\prime \prime}\right)$ with a 3-colorable triangulation as shown in Figure 3(a). (Figure 3(a) shows two iterations of this extension.) We can repeat this extension an arbitrary number of times; with each iteration this increases both, the number of non-pointed vertices and the overall number of vertices, by 3. It is easy to see that the resulting pseudo-triangulation $T_{r}(S)$ is 3 -colorable if and only if $T^{\prime \prime}\left(S^{\prime \prime}\right)$ is 3 -colorable.

The final rank of $T_{r}(S)$ equals the number of points added to $S^{\prime \prime}$. Thus $r=|S|-\left|S^{\prime \prime}\right|=n-\left|S^{\prime \prime}\right|$. For the reduction, we need not be able to compute $f(n)$, since we know that $f(n)$ is bounded from below by $c n^{\varepsilon}$, for a constant $c$ and $n$ larger than some constant $n_{0}$. To ensure that $r \geq i(S)-f(n)$, we add points to make $r=n-\left|S^{\prime \prime}\right| \geq i(S)-c n^{\varepsilon}$. Since $i(S)=n-3$, we have $\left|S^{\prime \prime}\right| \leq c n^{\varepsilon}+3$, and thus we can choose $n \in O\left(\left|S^{\prime}\right|^{1 / \varepsilon}\right)$. Therefore, the construction can be done by adding a polynomial number of points. The stated bound for the rank of $T_{r}(S)$ and NP-completeness follow.

Note that the construction applied in the proof above always gives a rank that is a multiple of 3 . However, removing one or two edges (shown bold in Figure 3(a)) in the final layer results in an arbitrary rank within the stated bounds. This shows that we can actually obtain any value for the difference between the rank and the maximum rank (even though this is not relevant for the reduction).

\section{Constant Maximum Face Degree}

In the previous section, NP-completeness of PT-3col turned out to be quite resistant against restrictions on the rank of pseudo-triangulations. Only for triangulations (which have maximal rank) and for pseudo-triangulations of very 
high rank (at least $i(S)-\Theta(\log n)$ ) do we know that the PT-3col problem is polynomial-time decidable.

In this section, we will consider pseudo-triangulations with constant maximum face degree. The degree of a face is defined as the number of its vertices. (The face degree should not be confused with the degree of a vertex, which is the number of its incident edges.) Note that we restrict the face degree of the pseudo-triangles, but not the degree of the outer face. The following two statements will allow us to transform any pseudo-triangulation with high maximal face degree into one with smaller maximal face degree, while still keeping rank and colorability properties.

Lemma 3. Any pseudo-triangle with $k>5$ vertices can be subdivided into two pseudo-triangles of degree strictly less than $k$, by adding an interior vertex of degree 2 such that pointedness of the involved vertices persists.

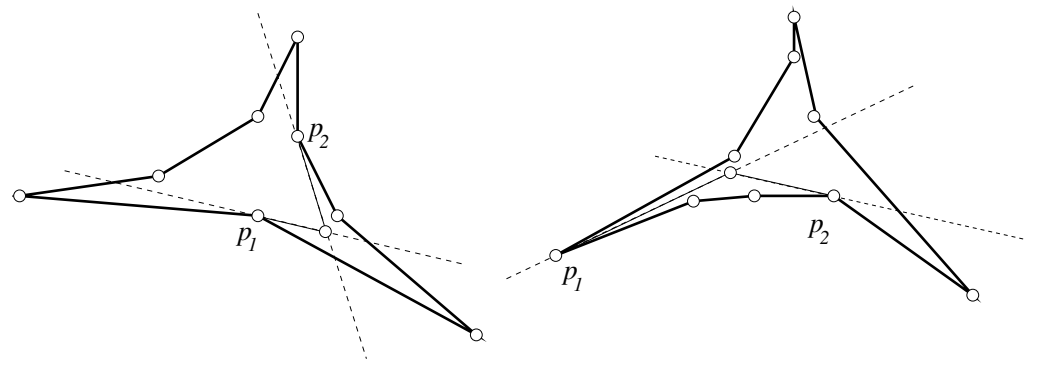

Figure 4: Subdividing large pseudo-triangles.

Proof. Let $\nabla$ be a pseudo-triangle of degree $k>5$. Choose an arbitrary vertex $p_{1}$ of $\nabla$ and another vertex $p_{2}$ of $\nabla$ which, when walking along the boundary of $\nabla$, is at least 3 edges away from $p_{1}$. In other words, along the side chains of $\nabla$ there are at least two vertices between $p_{1}$ and $p_{2}$; see Figure 4 for two examples. For both, $p_{1}$ and $p_{2}$, we consider an inner tangent at $p_{i}$ if $p_{i}$ is a reflex vertex in $\nabla$, or an angle bisector if $p_{i}$ is a convex vertex of $\nabla$. These two lines intersect inside $\nabla$ and we place a point $p_{\text {new }}$ at the intersection. Connecting $p_{\text {new }}$ to $p_{1}$ and $p_{2}$ now results in a subdivision of $\nabla$ into two pseudo-triangles of degree less than $k$, where $p_{\text {new }}$ has two incident edges, and all involved vertices are pointed.

Note that it may be necessary to slightly perturb $p_{\text {new }}$ if this vertex causes collinearities with the vertex set $S$ that spans $\nabla$. This will keep the general position assumption on $S \cup\left\{p_{\text {new }}\right\}$.

Corollary 2. Any pseudo-triangulation $T(S)$ with maximum face degree $k>5$ can be transformed, in polynomial time, into a pseudo-triangulation $T^{\prime}\left(S^{\prime}\right)$ with maximum face degree 5 and such that:

- $S \subseteq S^{\prime}$ and $\left|S^{\prime}\right|=\Theta(n)$, 
- $T(S)$ is 3-colorable if and only if $T^{\prime}\left(S^{\prime}\right)$ is 3-colorable,

- the rank of $T^{\prime}\left(S^{\prime}\right)$ equals the rank of $T(S)$.

Proof. Using Lemma 3, we can iteratively subdivide pseudo-triangles of degree $k>5$ in $T(S)$, without changing the rank. After adding $i=k-5$ vertices in such a pseudo-triangle, it gets subdivided with $2 i$ interior edges into $i+1$ pointed pseudo-triangles of degree 5 . Thus, using a linear number of new vertices, we obtain a pseudo-triangulation $T^{\prime}\left(S^{\prime}\right)$ with maximum face degree 5 . In each step, the added vertex has degree 2 , and we can easily find a proper 3 -coloring for $T^{\prime}\left(S^{\prime}\right)$ if we start with a proper 3-coloring of $T(S)$. As $T(S)$ is a subgraph of $T^{\prime}\left(S^{\prime}\right)$, the other direction follows trivially, and thus $T(S)$ is 3-colorable if and only if $T^{\prime}\left(S^{\prime}\right)$ is 3 -colorable.

\subsection{Face degree at most five}

In this subsection, we show that deciding 3-colorability remains NP-complete for pseudo-triangulations even if all inner faces have maximum degree 5. Moreover, NP-completeness persists for certain restrictions of the rank: If the problem is defined for pseudo-triangulations with a rank within $i(S)-\Omega\left(n^{\varepsilon}\right)$, for any constant $0<\varepsilon \leq 1$, the problem remains NP-complete.

We start with combining Corollary 2 with the NP-completeness result for PPT-3col in Theorem 1.

Corollary 3 (Face Degree $\leq \mathbf{5}$ Pointed Pseudo-Triangulation 3-Colorability, 5PPT-3col). Deciding whether a pointed pseudo-triangulation with maximum face degree 5 is 3-colorable is NP-complete.

Combining Corollary 3 with the construction used to prove the NP-completeness of $\mathrm{PT}_{r}-3 \mathrm{col}$ allows us to prove the following more general statement.

Theorem 4 (Face Degree $\leq \mathbf{5}$ Rank $\boldsymbol{r}$ Pseudo-Triangulation 3-colorability, $\left.5 \mathrm{PT}_{\boldsymbol{r}}-3 \mathrm{col}\right)$. For any constant $0<\varepsilon \leq 1$, let $f(x) \in \Omega\left(x^{\varepsilon}\right)$. Then it remains $N P$-complete to decide 3-colorability of a pseudo-triangulation $T(S)$ with maximum face degree 5 and with rank $r \geq i(S)-f(|S|)$.

Proof. The proof follows the lines of the proof of Theorem 3. The only difference is that we show the reduction from 5PPT-3col, and maintain the face degree $\leq 5$ property during the construction.

We start by circumscribing a pointed pseudo-triangulation with maximal face degree $5, T^{\prime}\left(S^{\prime}\right)$, with a triangle, $\triangle$. Applying Lemma 1 and Corollary 2, we can extend $T^{\prime}\left(S^{\prime}\right) \cup \triangle$ to a face degree $\leq 5$ pointed pseudo-triangulation $T^{\prime \prime}\left(S^{\prime \prime}\right)$, such that $\left|S^{\prime \prime}\right|=O\left(\left|S^{\prime}\right|\right)$ and $T^{\prime}\left(S^{\prime}\right)$ is 3-colorable if and only if $T^{\prime \prime}\left(S^{\prime \prime}\right)$ is 3 -colorable.

The bound for the rank can be obtained in the same way as for Theorem 3 .

For the sake of completeness, we state the following corollary. 
Corollary 4 (FACE Degree $\leq \mathbf{5}$ Pseudo-Triangulation 3-COLORABILITY, 5PT-3col). Deciding whether a pseudo-triangulation with maximum face degree 5 is 3-colorable is NP-complete.

\subsection{Face degree at most four}

In the proofs in the previous subsection, it was essential that pseudo-triangles of degree larger than 5 can always be subdivided. This result can not be extended to smaller pseudo-triangles. In fact, the situation changes completely if we bound the face degree of a pseudo-triangle by 4 .

Theorem 5. Pointed pseudo-triangulations with maximum face degree 4 are 3-colorable.

Proof. To prove this assertion, we use the concept of combinatorial pointed pseudo-triangulations (see [11]). These are embeddings of pointed pseudotriangulations where the edges need not to be straight lines, and pointedness is not a geometric property anymore. Instead, each vertex has a mark in one incident face, namely the one where it is pointed, and for each face all but three vertices have marks in this face. The only exception is the outer face, where all incident vertices have their mark in. Any given combinatorial pointed pseudotriangulation has a straight-line embedding such that every angle with a mark is reflex and all other angles are convex (see Section 5.2 in [7]). Thus, with respect to 3-colorability, combinatorial pointed pseudo-triangulations are equivalent to 'geometric' pointed pseudo-triangulations.
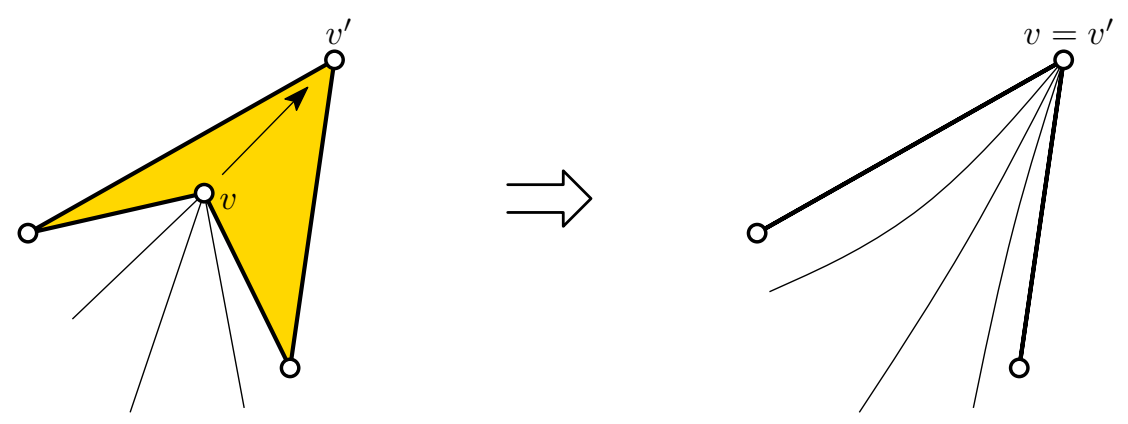

Figure 5: Merge operation to collapse a pseudo-triangle.

We now define a merge operation for an inner vertex $v$. It identifies $v$ with the antipodal convex vertex $v^{\prime}$ in the pseudo-triangle $\nabla$ it is pointed to, by 'moving' $v$ towards $v^{\prime}$; see Figure 5 . In this way $\nabla$ collapses, but, as will be discussed in detail below, the remaining graph is still a valid combinatorial pointed pseudo-triangulation with one vertex, one face, and two edges less.

We iterate this process as long as we have interior vertices. This can be done, as each such vertex is always pointed towards an interior pseudo-triangle of degree four. We claim that, after each such merging step, we again obtain 

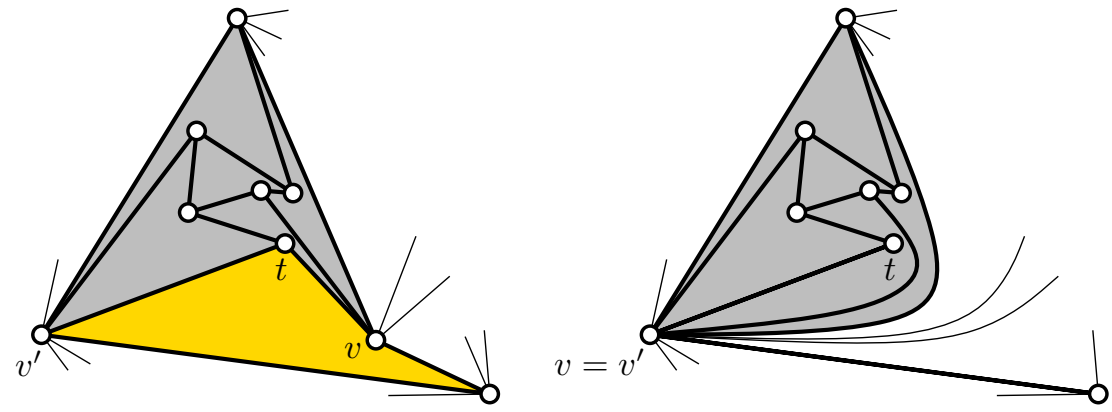

Figure 6: To avoid multiple edges, the vertices inside the gray region have to be merged prior to $v^{\prime}$.

a combinatorial pointed pseudo-triangulation; in particular, the resulting graph has to be simple, implying that we have to avoid loops (i.e., edges starting and ending in the same vertex) as well as multiple edges between vertex pairs. Further, all vertices of a pseudo-triangle need to be different (i.e., the pseudotriangle is non-degenerate).

Whenever a merging step would result in multiple edges (as shown in Figure 6), the vertices inside the cycle that would cause the multiple edge pair (i.e., vertices in the gray region in Figure 6) are merged prior to other vertices. Observe that, since there is a clear hierarchy on which step precedes another one, eventually vertex $t$ in Figure 6 will have degree two and no other vertex is inside the cycle; thus, $t$ can be merged. Apart from preventing multiple edges, we will show that degenerate pseudo-triangles cannot occur in any iteration by proving that there are no cycles in the merging steps. Below, we will argue in a similar way that there cannot be loops.

Due to the invariant that we always have a valid combinatorial pointed pseudo-triangulation, no inner vertices are left after all merging steps, and we obtain a (combinatorial) triangulation of a vertex set in convex position. The vertices of this triangulation are of course 3 -colorable, and we can assign their colors in linear time.

We finally invert the above process, and replicate in each reversed merge step the color of the original vertex to the duplicated vertex. This keeps the 3 -coloring valid, as these vertices have not been connected in the original graph. After all merge steps are undone, we obtain a proper 3-coloring of the given pointed pseudo-triangulation.

It remains to prove that we actually obtain a combinatorial pointed pseudotriangulation. The fact that we cannot obtain a degenerate pseudo-triangle in the graph resulting from a merging step follows from the observation that there are no cycles in the merging steps: Consider an abstract directed graph $G$ that is defined on the vertex set of the given pointed pseudo-triangulation $T$. $G$ contains an edge from $v$ to $v^{\prime}$ iff $v$ is reflex in the pseudo-triangle $\nabla$ and $v^{\prime}$ is its antipodal convex vertex. Because the antipodal convex vertex is unique for each pointed 
inner vertex, no vertex in $G$ has more than one outgoing edge. We show that $G$ is a forest with the outer vertices of $T$ being sinks. Suppose there is a cycle $C$ in $G$. We can actually draw $C$ on the pseudo-triangulation $T$ such that the resulting graph $C \cup T$ is still plane. This splits $|C|$ quadrilaterals of $T$ into $2|C|$ triangles; half of them are inside $C$. We consider the subgraph of $T$ in the interior of $C$ plus $C$ itself as a combinatorial pointed pseudo-triangulation $T_{C}$ with arbitrary pointedness marks for the vertices on $C$. Clearly, $T_{C}$ has maximum face degree 4 , has $|C|$ outer vertices, and contains at least $|C|$ triangles. A combinatorial pointed pseudo-triangulation with maximum face degree 4 that has $b$ outer vertices of which $r$ are reflex is known to contain exactly $\delta=b-2 r-2$ triangles (see Corollary 1 in [4]). However, because for $T_{C}$ we have $b=|C|, T_{C}$ may only contain at most $|C|-2$ triangles, a contradiction. Hence, $G$ is a forest and we therefore cannot obtain a degenerate pseudo-triangle.

Finally, suppose merging two vertices $v$ and $v^{\prime}$ sharing a pseudo-triangle $\nabla$ would lead to a loop in the resulting graph. Then $v$ and $v^{\prime}$ would have to be connected by an edge, which is outside $\nabla$. This edge forms two 3-cycles with the edges of $\nabla$. However, one of the other vertices of $\nabla$ is pointed towards the inside of the 3-cycle, contradicting the fact that the previous graph was a combinatorial pointed pseudo-triangulation.

Observe that the proof above provides a linear-time algorithm for finding a 3 -coloring. The obtained 3-coloring is special, in the sense that for every inner face of degree 4 of the pointed pseudo-triangulation, the reflex vertex has the same color as its antipodal convex vertex. In fact, up to permutation of the three colors, there is only one coloring with this property. This follows from the facts that (1) a 3-coloring of a triangulation of a convex point set is unique, up to permutation, and (2) the merge steps used in the above proof lead to a unique triangulation of the convex set, independent of their order.

Note further that the merge step will not work for pseudo-triangles of degree larger than 4 . We would obtain inner faces with only two vertices which are not marked as pointed in this face, and thus the resulting graph would not be a valid combinatorial pointed pseudo-triangulation. Therefore, not all inner vertices can be reduced with the merge operation, and we end up with a triangulation of a general point set. As mentioned, we can decide in linear time whether there exists a 3-coloring for this triangulation. In the affirmative case this also induces a proper 3-coloring of the initial pointed pseudo-triangulation. But in the negative case, we cannot conclude that such a coloring does not exist, as different orders of reduction steps might lead to a different triangulation, which still could be 3 -colorable.

In the special case of pointed pseudo-triangulations with maximal face degree 4, living on a vertex set with a convex hull of size at most 5 , we can alternatively derive 3-colorability from the following theorem by Grünbaum.

Theorem 6 (First part of Grünbaum's Theorem[13]). If $G$ is a planar graph with at most three 3-cycles, then $G$ is 3-colorable. 
Namely, for a vertex set with a convex hull of size 3,4 , or 5 , a pointed pseudotriangulation $T$ contains 1,2 , or 3 triangles (faces of degree 3 ), respectively. If, in addition, there exist induced triangles (i.e., non-empty 3 -cycles) in $T$, then exactly one (empty) triangle of $T$ has to be contained in each of them. When removing interior parts of induced triangles, we obtain a graph that satisfies the conditions of Grünbaum's Theorem. Recursively, this applies also to the removed subgraphs, and 3-colorability of $T$ follows.
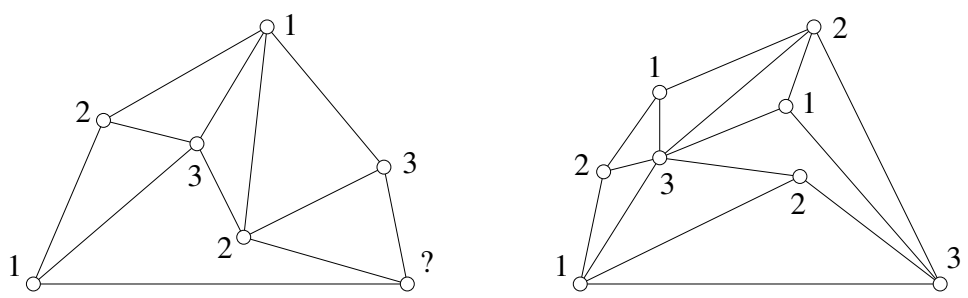

Figure 7: Two pseudo-triangulations only one of which can be 3-colored.

Pointed pseudo-triangulations with maximum face degree 4 thus are a special structure concerning 3 -colorability. Note that triangulations of convex point sets also fall into this class. Investigating the influence on 3-colorability of the rank of pseudo-triangulations in that class reveals that already a rank of 1 allows pseudo-triangulations which are not 3-colorable; one example would be a plane drawing of $K_{4}$, a more general example is exhibited in Figure 7 (left). Note that all inner vertices in the graph shown have even degree. Hence, this parity property, which can be used to prove 3-colorability for triangulations, does not carry over to general rank pseudo-triangulations. Moreover, there exist examples (see Figure 7, right) with non-pointed inner vertices of odd degree, which are still 3-colorable.

In the remainder of this section, we will prove NP-completeness for a wide range of ranks for maximum face degree 4 pseudo-triangulations. More specifically, we show that the problem remains NP-complete as long as the interval of allowed ranks overlaps with $i(S)-\Omega\left(n^{\varepsilon_{1}}\right)$ and $\Omega\left(n^{\varepsilon_{2}}\right)$, which is formalized in the same way as in Theorem 3 .

Theorem 7 (Face Degree $\leq 4$ Rank $\boldsymbol{r}$ Pseudo-Triangulation 3-colorability, $\left.4 \mathrm{PT}_{\boldsymbol{r}}-3 \mathrm{col}\right)$. Deciding whether a pseudo-triangulation with maximum face degree 4 is 3-colorable is NP-complete. For any two constants $0<\varepsilon_{1}, \varepsilon_{2} \leq 1$, let $f(x) \in \Omega\left(x^{\varepsilon_{1}}\right)$ and $g(x) \in \Omega\left(x^{\varepsilon_{2}}\right)$. Then it remains $N P$-complete to decide 3-colorability of a pseudo-triangulation $T(S)$ with maximum face degree 4 and with rank $r \geq i(S)-f(|S|)$ or $r \leq g(|S|)$.

Proof. Like for Theorem 4, we prove NP-completeness by reduction from 5PPT$3 \mathrm{col}$ and start with a face degree $\leq 5$ pointed pseudo-triangulation, $T^{\prime}\left(S^{\prime}\right)$. We circumscribe $T^{\prime}\left(S^{\prime}\right)$ with a triangle and, by applying Lemma 1 and Corollary 2 , obtain a face degree $\leq 5$ pointed pseudo-triangulation $T^{\prime \prime}\left(S^{\prime \prime}\right)$, such that 
$\left|S^{\prime \prime}\right|=O\left(\left|S^{\prime}\right|\right),\left|C H\left(S^{\prime \prime}\right)\right|=3$, and $T^{\prime}\left(S^{\prime}\right)$ is 3-colorable if and only if $T^{\prime \prime}\left(S^{\prime \prime}\right)$ is 3 -colorable.
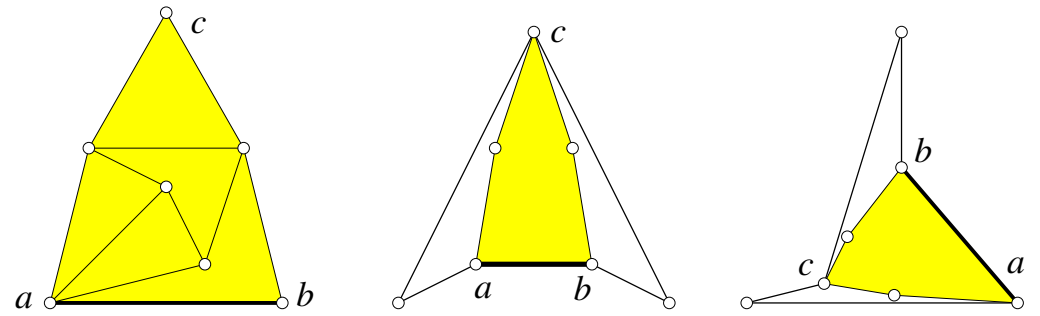

Figure 8: Gadget for the proof of Theorem 7 (left), and the two ways of splitting a pseudo-triangle of size 5 (middle and right).

We next show how to reduce a pseudo-triangle $\nabla$ of degree 5 in $T^{\prime \prime}\left(S^{\prime \prime}\right)$ to 7 pseudo-triangles of maximum degree 4 , such that the resulting graph will be 3 -colorable if and only if $T^{\prime \prime}\left(S^{\prime \prime}\right)$ is 3-colorable. To this end, we use the gadget shown in Figure 8 (left). It connects an edge $\overline{a b}$ of $\nabla$ to an opposite vertex $c$ of $\nabla$. Note that whatever (different) colors the vertices $a$ and $b$ get assigned, we can choose for $c$ either one of the colors from $a$ or $b$ or the remaining third color, and obtain in all cases a proper 3-coloring of the gadget. (The previously used gadgets would not allow us to further reduce the face degree.)

There are two different types of pseudo-triangles of degree 5 . For the first type, one side-chain contains 4 vertices, and we connect the middle edge of this side-chain to the antipodal convex vertex of $\nabla$; see Figure 8 (middle). For the second type, there are two side-chains containing 3 vertices and a side-chain consisting of a single edge. In this case, the two reflex vertices of $\nabla$ can 'see' each other in the interior of $\nabla$. Thus, for at least one of the two longer sidechains, the edge incident to the short side-chain can always be connected to the opposite reflex vertex by our gadget; see Figure 8 (right).

In this way, we add a gadget for each pseudo-triangle of degree 5 in $T^{\prime \prime}\left(S^{\prime \prime}\right)$. This results in a face degree $\leq 4$ pseudo-triangulation, $T^{*}\left(S^{*}\right)$, at the expense of pointedness, as we increase the rank with each added gadget by 2 . The entire construction can be done in polynomial time. We have $\left|S^{*}\right|=\Theta\left(\left|S^{\prime}\right|\right)$, the convex hull is still a triangle, and $T^{\prime}\left(S^{\prime}\right)$ is 3 -colorable if and only if $T^{*}\left(S^{*}\right)$ is 3 -colorable.

Extending $T^{*}\left(S^{*}\right)$ with a 3-colorable triangulation as shown in Figure 3(a) increases the number of non-pointed vertices, and thus the rank, with each added vertex. As in the proof of Theorem 3, we can reach a rank of $r \geq i(S)-c n^{\varepsilon_{1}} \geq$ $r^{*}$ by adding $O\left(\left|S^{\prime}\right|^{1 / \varepsilon_{1}}\right)$ vertices, with $r^{*}$ being the rank of $T^{*}\left(S^{*}\right)$, for any constant $c$ in the bound of $f(n)$.

For low rank we use a different extension, as $r^{*}$ can be of size $\Theta\left(\left|S^{*}\right|\right)$. The 3-colorable face degree $\leq 4$ pseudo-triangulation depicted in Figure 3(b) increases the number of pointed vertices with each added vertex. By adding $O\left(\left|S^{\prime}\right|^{1 / \varepsilon_{2}}\right)$ vertices using this construction, we can reach a rank of $r=r^{*} \leq c n^{\varepsilon_{2}}$, 
for any constant $c$ in the bound of $g(n)$.

After the two extensions described, the emerging face degree $\leq 4$ pseudotriangulation is 3 -colorable if and only if the initial face degree $\leq 5$ pointed pseudo-triangulation is 3-colorable. Thus, NP-completeness follows for rank- $r$ pseudo-triangulations with maximal face degree 4 , for any rank within the given bounds.

Note that in the proof above, two vertices become non-pointed whenever the gadget of Figure 8 (left) is added. Therefore, our reduction does not work for pointed pseudo-triangulations.

\section{Further Research}

We have presented several complexity results on the colorability of pseudotriangulations. For the sake of clarity they will be listed again below, followed by some open problems raised in this context.

The following assertions on pseudo-triangulations of maximum face degree 4 have been proved:

- rank 0 (pointed pseudo-triangulations): always 3-colorable.

- rank $r, \Theta\left(n^{\varepsilon_{2}}\right) \leq r \leq i(S)-\Theta\left(n^{\varepsilon_{1}}\right)$ : NP-complete.

- rank $r, i(S)-\Theta(\log n) \leq r \leq i(S)$ : decidable in polynomial time.

Moreover, for rank- $r$ pseudo-triangulations of maximum face degree 5 , and rank- $r$ pseudo-triangulations without any face degree bound, 3-colorability is NP-complete as long as $r \leq i(S)-\Theta\left(n^{\varepsilon}\right)$. On the other hand, for both classes, 3 -colorability is decidable in polynomial time, provided $r \geq i(S)-\Theta(\log n)$.

One interesting question left open is where precisely the changes between 'NP-complete' and 'linear-time decidable' take place. Furthermore, what can be said if a pseudo-triangulation of maximum face degree 4 is 'almost pointed', i.e., has rank $c$ for some small integer constant $c \geq 1$ ?

Another open problem, which draws connections between 3-colorability, adding Steiner points for even parity, and Abam's problem (Problem 9 in [10]), is the following: Let $S$ be a set of $n$ points in the plane in general position. What is the minimum number $k$, such that for any $S$ there exists a subset $S^{\prime} \subset S$ with cardinality at least $n-k$, which can be triangulated in a way that any interior vertex of $S^{\prime}$ has even degree?

In other words, how many vertices do we have to remove from $S$ such that we can guarantee the existence of a 3 -colorable triangulation for the remaining set? 


\section{Acknowledgments}

This work was initiated during the Sixth European Pseudo-Triangulation Working Week in Ratsch an der Weinstraße, Austria, September 2009. We would like to thank the participants of the workshop for stimulating discussions.

A preliminary version appeared in conference proceedings.[3] O. A. and A. P. are supported by the ESF EUROCORES programme EuroGIGA - ComPoSe, Austrian Science Fund (FWF): I 648-N18. F. A. supported by the ESF EUROCORES programme EuroGIGA - Voronoi, Austrian Science Fund. T. H. supported by the FWF grant P23629-N18. C. H. supported by projects MINECO MTM2012-30951 and Gen. Cat. DGR 2014SGR46. Part of this work has been done while O. A., F. A., and B. V. were supported by the FWF [Austrian Fonds zur Förderung der Wissenschaftlichen Forschung] grant S9205-N12, NFN Industrial Geometry, and while A. P. was recipient of a DOC-fellowship of the Austrian Academy of Sciences at the Institute for Software Technology, Graz University of Technology, Austria.

\section{References}

[1] O. Aichholzer, F. Aurenhammer, P. Brass, H. Krasser. Pseudotriangulations from surfaces and a novel type of edge flip. SIAM Journal on Computing, 32:1621-1653, 2003.

[2] O. Aichholzer, F. Aurenhammer, P. Gonzalez-Nava, T. Hackl, C. Huemer, F. Hurtado, H. Krasser, S. Ray, B. Vogtenhuber. Matching edges and faces in polygonal partitions. Computational Geometry, Theory and Applications, 39:134-141, 2008.

[3] O. Aichholzer, F. Aurenhammer, T. Hackl, C. Huemer, A. Pilz, B. Vogtenhuber. 3-Colorability of pseudo-triangulations. In Proc. 26th European Workshop on Computational Geometry, Dortmund, Germany, 2010, 2124 .

[4] O. Aichholzer, T. Hackl, D. Orden, A. Pilz, M. Saumell, B. Vogtenhuber. Flips in combinatorial pointed pseudo-triangulations with face degree at most four. International Journal of Computational Geometry \& Applications, 24:197-224, 2014.

[5] K. Diks, L. Kowalik, M. Kurowski. A new 3-color criterion for planar graphs. In L. Kučera (ed.), Graph-Theoretic Concepts in Computer Science, Lecture Notes in Computer Science, 2573:138-149, Springer, 2002.

[6] M. N. Ellingham, H. Fleischner, M. Kochol, and E. Wenger. Colorability of planar graphs with isolated nontriangular faces. Graphs and Combinatorics, 20:443-446, 2004. 
[7] R. Haas, D. Orden, G. Rote, F. Santos, B. Servatius, H. Servatius, D. Souvaine, I. Streinu, W. Whiteley. Planar minimally rigid graphs and pseudotriangulations. Computational Geometry, Theory and Applications, 31:3161,2005 .

[8] T.R. Jensen and B. Toft. Graph Coloring Problems. Wiley Series in Discrete Mathematics and Optimization, 1994.

[9] M. Kochol. Linear algorithm for 3-coloring of locally connected graphs. In: K. Jansen et al. (eds.), Experimental and Efficient Algorithms, Lecture Notes in Computer Science 2647:191-194, Springer, 2003.

[10] J. S. Mitchell. Open Problem Session. In: P. K. Agarwal et al. (eds.), Computational Geometry, Dagstuhl Seminar Proceedings 09111, Schloss Dagstuhl - Leibniz-Zentrum fuer Informatik, Germany, 2009.

[11] D. Orden, F. Santos, B. Servatius, H. Servatius. Combinatorial pseudotriangulations. Discrete Mathematics, 307:554-566, 2007.

[12] G. Rote, F. Santos, I. Streinu, Pseudo-triangulations - a Survey. Contemporary Mathematics, 453:343-410, 2008.

[13] R. Steinberg. The state of the three color problem. In J. Gimbel, J. W. Kennedy, and L. V. Quintas (eds.), Quo vadis, graph theory? A source book for challenges and directions, Annals of Discrete Mathematics, $55: 211-248,1993$.

[14] L. Stockmeyer. Planar 3-colorability is polynomial complete. SIGACT News, 5:19-25, 1973.

[15] I. Streinu. A combinatorial approach to planar non-colliding robot arm motion planning. In Proc. 41st Ann. IEEE Symp. on Foundations of Computer Science, 2000, 443-453. 